\title{
26. SEDIMENTS AND THEIR GEOCHEMISTRY, HESS DEEP ${ }^{1}$
}

\author{
J.-D. Eckhardt ${ }^{2}$ and H. Puchelt ${ }^{2}$
}

\begin{abstract}
Sediments from Hess Deep, Ocean Drilling Program Leg 147 Site 894, and from the deepest point of Hess Deep (recovered during cruise Sonne 60, core 95) were examined. Different lithologies within these sediments have different geochemical signatures. It is possible to distinguish the source rocks of the different sediment types and to reconstruct the processes affecting the genesis of the sediments. Foraminiferal ooze from Site 894 has high $\mathrm{Sr}$ concentrations and elevated but not constant Ba values. Sediments from lithic breccia sequences have nearly the same element concentrations and rare earth element (REE) patterns as underlying gabbroic rocks. The lithic breccias are a pure physical weathering product, exhibiting no alteration. Hydrothermal sediments from core 95 have varying concentrations of $\mathrm{Mn}$, As, and other elements precipitated by hydrothermal activity. A serpentine sequence in the middle of the sediment-core is of turbiditic origin, inferred from high $\mathrm{Mg}, \mathrm{Ni}$, and $\mathrm{Cr}$ concentrations. Platinum group elements (PGE) in the serpentinitic sediments were fractionated during the alteration of peridotite. In the hydrothermal sediments PGE are partially enriched.
\end{abstract}

\section{INTRODUCTION}

The aim of Leg 147 was to investigate the evolution of crustal processes and the internal stratigraphy of oceanic crust and mantle at a fast-spreading ridge. The chemical effects of serpentinization are another aspect of these investigations. The geochemistry of sediments might play a role in understanding these processes.

The recovery of sediments was not the goal of this leg. Sediments were only recovered from thickness probes to locate a minimal sediment cover to place the guide base. Video images showed a thin widespread blanket of sediments in the working area.

Sediments were recovered in short cores from Holes 894A, 894D, and $894 \mathrm{E}$ (Fig. 1) and as a perturbed section in Hole 895A. The sediments at Site 894 consist of pelagic ooze with foraminifers, basaltic cobbles, basaltic lithic breccias, basaltic sand, and foraminiferal sand. At Hole 895A foraminiferal nannofossil clay with serpentine and gabbro clasts was recovered.

From pre-site survey cruise Sonne 60 we obtained the sedimentbox core 95 from the deepest point of Hess Deep (Fig. 1). The sediments consist of a pelagic sequence affected by hydrothermal activity that was interrupted by a serpentinitic sequence (Eckhardt, 1993).

Investigations on sediments in Hess Deep are of interest because of their direct genetic relation to the outcropping altered rocks. Geochemistry of the sediments reflects the behavior of elements (e.g., Ni, REE, PGE) during alteration, influence of hydrothermal activity, and the role of other processes such as circulation of seawater.

\section{ANALYTICAL METHODS}

All sediments were leached by dialysis to remove seawater salt. After sieving, geochemical investigations were made on grains finer than $60 \mu \mathrm{m}$. Mineral phases were analyzed by X-ray diffractometry (XRD) (Siemens D500) and differential thermal analysis-thermogravimetry (DTA-TG). Carbon and sulfur content was determined by infrared detection (Leybold Heraeus CSA 302). Detection limits are $100 \mathrm{ppm}$ for sulfur and $500 \mathrm{ppm}$ for carbon. Discrimination of organ-

'Mével, C., Gillis, K.M., Allan, J.F., and Meyer, P.S. (Eds.), 1996. Proc. ODP, Sci. Results, 147: College Station, TX (Ocean Drilling Program).

${ }^{2}$ Institut für Petrographie und Geochemie der Universität Karlsruhe, Kaiserstraße 12, D-76128 Karlsruhe, Federal Republic of Germany.

detlef.eckhardt@bio-geo.uni-karlsruhe.de

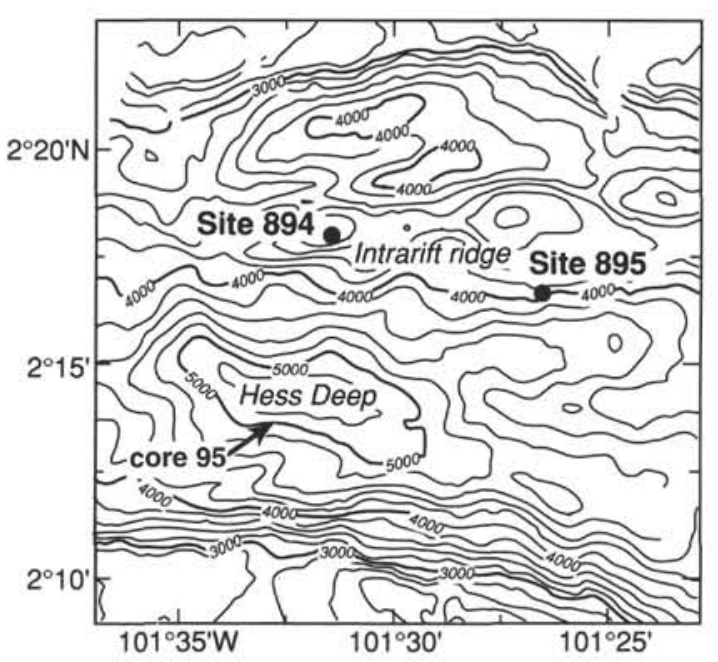

Figure 1. Location of Sites 894, 895, and core 95 at Hess Deep. Bathymetric contours from Sonne data (Cruise report, Sonne 60).

ic carbon and carbonate was achieved by treatment with hydrochloric acid and analysis of remaining carbon in comparison to total carbon.

Concentrations of major elements in core 95 were analyzed by X-ray fluorescence (XRF) (Philips PW 1450-20, on melt-pellets). Trace elements were analyzed by energy-dispersive X-ray fluorescence analysis (XRFA) and instrumental neutron activation analysis. XRFA was carried out using a "Tracor Xray Spectrace 430 " $(1 \mathrm{~mA} /$ $50 \mathrm{kV}$ Rh-tube and a Si[Li] detector [ $30 \mathrm{~mm}^{2}$ active area] with an energy resolution of $145 \mathrm{eV}$ at $6.4 \mathrm{keV})$. Neutron activation was performed at TRIGA II (University of Heidelberg), which has a neutron flux of $8 \times 10^{12} \mathrm{~cm}^{-2} \mathrm{~s}^{-1}$. Duration of irradiation was $5 \mathrm{hr}$. The samples were analyzed with two detectors: one for low energy (planar hpGedetector [30-210 keV] with an energy resolution of $570 \mathrm{eV}$ at 122 $\mathrm{keV}$ ) and one for high energy (coaxial hpGe detector [150-2000 keV] with an energy resolution of $1.95 \mathrm{keV}$ at $1.332 \mathrm{MeV}$, efficiency $15 \%$ ).

Platinum group elements (PGE) were evaluated by nickel sulfide fire-assay for 14 of the 38 core 95 samples. PGE were brought into solution and detected by inductively coupled plasma-mass spectrometry (ICP-MS) (Fisons Plasmaquad Turbo $2+$ ). Because $25 \mathrm{~g}$ is the minimum for analysis of PGE, this could not be done on sediment samples of Leg 147. 


\section{SAMPLE DESCRIPTION}

The sediments from Site 894 were recovered using the drill string, while testing the thickness of the sediment cover. Sediment thickness in this area is about $3.4 \pm 0.9 \mathrm{~m}$. Site 894 is located at the western margin of the Equatorial Upwelling Zone. Here we find high bioproductivity with fast accumulation of microorganisms and their calcareous and siliceous shells. The carbonate compensation depth (CCD) in this region is about $4 \mathrm{~km}$ (Horn et al., 1973). The holes at Site 894 are located above CCD, whereas the bottom of Hess Deep (core 95, Sonne 60 ) is located below this level.

Sediments from Leg 147 were sampled at almost regular intervals downcore, and changes in lithology were considered. Core 95 was sampled in 10-cm intervals downcore; between 350 and $360 \mathrm{~cm}$ an additional sample was taken.

\section{Hole 894A}

Hole $894 \mathrm{~A}$ had a recovery of more than $6 \mathrm{~m}$ of sediment, mostly disturbed by drilling. The carbonate content decreases in the upper 1 $\mathrm{m}$ from $27.1 \% \mathrm{CO}_{2}$ at $22 \mathrm{~cm}$ depth to $11.7 \% \mathrm{CO}_{2}$ at $59 \mathrm{~cm}$ and about $3.7 \%$ at $1 \mathrm{~m}$. Below $1.79 \mathrm{~m}$, the values of $\mathrm{CO}_{2}$ vary between $0.2 \%$ and $1.3 \%$. This is to be expected as the uppermost $1 \mathrm{~m}$ of sediment consists of foraminiferal sand. Below $1 \mathrm{~m}$, we find an igneous lithic breccia with a grain size ranging from coarse sand to pebbles.

\section{Hole 894D}

About $1.3 \mathrm{~m}$ of sediment was recovered out of $10 \mathrm{~m}$ penetration. The sediments are foraminiferal sands and ooze, with a $\mathrm{CO}_{2}$ content of $31.1 \%$ and $31.9 \%$ in our two samples.

\section{Hole 894E}

The sediments of this hole have nearly the same lithology as found in Hole 894D. About $2.4 \mathrm{~m}$ of foraminiferal sand and ooze with brecciated parts of gabbros was recovered. Below $1.5 \mathrm{~m}$, two horizons of lithic breccia are intercalated.

\section{Core 95-Sonne 60}

Core 95, collected during cruise Sonne 60 , was taken from the deepest point of Hess Deep $\left(2^{\circ} 14.29^{\prime} \mathrm{N}, 101^{\circ} 32.88^{\prime} \mathrm{W}, 5410 \mathrm{mbsl}\right)$. Besides hydrothermal activity, the main factor controlling the sediment lithology is turbidites from surrounding slopes. The $360-\mathrm{cm}$ core is divided by color into two types of sediments. The top $57 \mathrm{~cm}$ is composed of dark brown sediments, the color due to iron oxidation. The middle part $(85-300 \mathrm{~cm})$ is grayish olive serpentine, and sequences above and below are dark olive ooze.

\section{RESULTS}

\section{Site 894}

Strontium and barium show a strong correlation with the carbonate content of the sediments (Table 1). Strontium is an indicator for calcite; barium is bound in a separate phase of marine precipitation, probably as baryte (Puchelt, 1967). Ba could also be in Mn-oxides or clay minerals. In the case of the sediments from Hess Deep, the specific $\mathrm{Ba}$ mineral could not be traced because the content is not high enough for XRD. Nevertheless, at Site 894 the Ba phase is a marine precipitate or an early diagenetic product and not concerned with processes like alteration or hydrothermal activity.

The REE patterns of the sediments plot in two distinctive areas (Fig. 2). Calcareous sediments are affected by the pattern of seawater with a negative $\mathrm{Ce}$ anomaly and an enrichment of the light REE. The slight negative europium anomaly results from a silicate component in the foraminiferal ooze.

The noncalcareous sediments have a pattern similar to gabbros or mid-ocean-ridge basalt (MORB) and have nearly the same content of REE. The concentrations of major and minor elements are also identical to gabbroic rocks, showing little, if any, alteration.

Table 1. Chemical composition of sediment samples, Site 894.

\begin{tabular}{|c|c|c|c|c|c|c|c|c|c|c|c|c|c|c|c|c|}
\hline \multirow[b]{2}{*}{$\begin{array}{l}\text { Core, section, } \\
\text { interval }(\mathrm{cm})\end{array}$} & \multirow[b]{2}{*}{$\begin{array}{l}\text { Depth } \\
\text { (mbsf) }\end{array}$} & \multicolumn{2}{|c|}{ CSA } & \multicolumn{6}{|c|}{ XRFA } & \multicolumn{7}{|c|}{ INAA } \\
\hline & & $\underset{(\%)}{S}$ & $\begin{array}{l}\mathrm{CO}_{2} \\
(\%)\end{array}$ & $\begin{array}{l}\mathrm{MnO} \\
(\%)\end{array}$ & $\begin{array}{c}\mathrm{Cu} \\
(\mathrm{ppm})\end{array}$ & $\underset{(\mathrm{ppm})}{\mathrm{Zn}}$ & $\begin{array}{c}\mathrm{Sr} \\
(\mathrm{ppm})\end{array}$ & $\begin{array}{c}\mathrm{Pb} \\
(\mathrm{ppm})\end{array}$ & $\begin{array}{c}\mathrm{Cr} \\
\text { (ppm) }\end{array}$ & $\begin{array}{c}\mathrm{Fe}_{2} \mathrm{O}_{3} \\
(\%)\end{array}$ & $\begin{array}{c}\text { Co } \\
(\mathrm{ppm})\end{array}$ & $\begin{array}{c}\mathrm{Ni} \\
(\mathrm{ppm})\end{array}$ & $\begin{array}{l}\mathrm{Zn} \\
(\mathrm{ppm})\end{array}$ & $\begin{array}{c}\mathrm{Rb} \\
(\mathrm{ppm})\end{array}$ & $\begin{array}{c}\mathrm{Sb} \\
(\mathrm{ppm})\end{array}$ & $\begin{array}{c}\mathrm{Ba} \\
(\mathrm{ppm})\end{array}$ \\
\hline \multicolumn{17}{|l|}{$147-894 \mathrm{~A}-$} \\
\hline IR-1, 22-27 & 0.22 & 0.06 & 27.2 & 0.21 & 85 & 92 & 1175 & $<10$ & 37 & 4.4 & 12.4 & 28 & 90 & 6 & 0.8 & 1709 \\
\hline $1 \mathrm{R}-1,59-61$ & 0.59 & 0.01 & 11.8 & 0.55 & 110 & 195 & 518 & 21 & 78 & 11.3 & 39.6 & 63 & 116 & 6 & 0.2 & 470 \\
\hline 1R-1, 97-99 & 0.97 & 0.02 & 0.9 & 0.28 & 56 & 98 & 101 & 11 & 124 & 14.5 & 51.1 & 64 & 97 & 7 & 0.3 & $<50$ \\
\hline $1 \mathrm{R}-1,128-130$ & 1.28 & 0.03 & 4.3 & 0.57 & 94 & 345 & 232 & 14 & 92 & 13.7 & 47.9 & 40 & 205 & 6 & 0.2 & 183 \\
\hline $1 \mathrm{R}-2,0-2$ & 1.50 & 0.03 & 3.2 & 0.42 & 85 & 152 & 189 & 12 & 91 & 13.9 & 49.0 & 73 & 135 & 8 & 0.2 & 135 \\
\hline |R-2, 29-31 & 1.79 & 0.03 & 0.1 & 0.33 & 62 & 154 & 84 & $<10$ & 84 & 14.9 & 50.7 & 38 & 125 & 7 & 0.3 & 65 \\
\hline $1 \mathrm{R}-2,61-63$ & 2.11 & 0.02 & 1.3 & 0.44 & 90 & 176 & 132 & 12 & 90 & 14.3 & 47.7 & 35 & 108 & 8 & 0.3 & 61 \\
\hline IR-2, 94-96 & 2.44 & 0.03 & 0.7 & 0.31 & 57 & 123 & 89 & $<10$ & 90 & 14.6 & 49.9 & 63 & 139 & 5 & 0.3 & $<50$ \\
\hline IR-3,3-5 & 3.03 & 0.17 & 1,1 & 0.53 & 104 & 256 & 130 & 16 & 89 & 14.5 & 48.4 & 66 & 132 & 10 & 0.3 & 67 \\
\hline IR-3, 26-28 & 3.26 & 0.07 & 0.9 & 0.82 & 124 & 365 & 132 & 22 & 90 & 14.5 & 49.3 & 59 & 161 & 5 & 0.3 & $<50$ \\
\hline IR-3, 79-81 & 3.79 & 0.08 & 0.4 & 0.29 & 40 & 119 & 83 & $<10$ & 82 & 14.7 & 48.5 & 67 & 105 & 9 & 0.3 & $<50$ \\
\hline $1 \mathrm{R}-3,132-134$ & 4.32 & 0.16 & 0.5 & 0.34 & 61 & 125 & 83 & $<10$ & 88 & 14.5 & 49.1 & 56 & 131 & 6 & 0.2 & $<50$ \\
\hline IR-4, 31-33 & 4.81 & 0.15 & 0.6 & 0.27 & 52 & 130 & 92 & $<10$ & 83 & 14.7 & 48.0 & 51 & 131 & $<2$ & 0.2 & 60 \\
\hline $1 \mathrm{R}-4,79-81$ & 5.29 & 0.27 & 0.2 & 0.27 & 60 & 123 & 83 & $<10$ & 83 & 14.5 & 48.0 & 35 & 123 & $<2$ & 0.3 & $<50$ \\
\hline IR-5, 7-9 & 5.57 & 0.20 & 0.6 & 0.33 & 67 & 147 & 100 & 10 & 93 & 14.1 & 46.5 & 44 & 125 & 9 & 0.3 & 51 \\
\hline IR-5, 54-56 & 6.04 & 0.06 & 0.3 & 0.27 & 48 & 111 & 81 & $<10$ & 81 & 14.3 & 46.3 & 45 & 111 & 10 & 0.1 & $<50$ \\
\hline \multicolumn{17}{|l|}{ 147-894D- } \\
\hline IR-1, 47-49 & 0.47 & 0.02 & 31.9 & 0.26 & 89 & 70 & 1377 & $<10$ & 26 & 2.0 & 6.2 & 20 & 49 & 2 & 0.7 & 1576 \\
\hline $1 \mathrm{R}-1,99-101$ & 0.99 & 0.04 & 31.1 & 0.62 & 138 & 144 & 1233 & 11 & 30 & 4.1 & 14.5 & 43 & 125 & 4 & 1.0 & 3084 \\
\hline \multicolumn{17}{|l|}{ 147-894E- } \\
\hline IR-1, 1-3 & 0.01 & 0.02 & 31.9 & 1.46 & 216 & 1145 & 1373 & 36 & 49 & 4.3 & 21.2 & 32 & 327 & $<2$ & 0.6 & 1350 \\
\hline IR-1, 50-52 & 0.50 & 0.06 & 31.5 & & & & & & 52 & 4.7 & 23.7 & 58 & 381 & $<2$ & 0.5 & 1364 \\
\hline IR-1,99-101 & 0.99 & 0.08 & 24.9 & & & & & & 66 & 6.3 & 29.7 & 54 & 399 & $<2$ & 0.6 & 1341 \\
\hline IR-I, 148-150 & 1.48 & 0.02 & 35.1 & 0.07 & 81 & 59 & 1329 & $<10$ & 20 & 2.6 & 11.5 & 26 & 46 & 5 & 0.5 & 1568 \\
\hline IR-2, 8-10 & 1.58 & 0.03 & 38.8 & 0.11 & 84 & 105 & 1390 & $<10$ & & & & & & & & \\
\hline IR-2, 47-49 & 1.97 & 0.06 & 37.4 & 0.37 & 80 & 88 & 1294 & $<10$ & & & & & & & & \\
\hline
\end{tabular}

Notes: $\mathrm{CSA}=$ carbon sulfur analyzer. $\mathrm{XRFA}=\mathrm{X}$-ray fluorescence analysis. INAA $=$ instrumental neutron activation analysis 


\section{Core 95}

The color division of the sediment core is reflected in major- and minor-element concentration of the sediments (Table 2). In Figure 3 we see that nickel concentration increases sharply at $85 \mathrm{~cm}$ and decreases at $300 \mathrm{~cm}$. This high Ni content is confirmed by mineralogical data, with chrysotile (detected by XRD and DTA-TG) as the main phase. Chrysotile is an alteration product of peridotites high in $\mathrm{Ni}$ (Moody, 1976; Cannat et al., 1992; Boillot et al., 1980; Bonatti et al., 1973) and forms by hydration of forsterite at temperatures below $350^{\circ} \mathrm{C}$ (Evans et al., 1976; Deer et al., 1982).

Major and trace elements indicate that silicates between 80 and $210 \mathrm{~cm}$ consist mainly of chrysotile. However, another alumosilicate phase must also be present, because there is a difference between the chemical composition of chrysotile and the total composition of the sediment (Table 3). This difference is consistent with the composition of nontronite found by mineralogical methods only in the upper parts of the core.

The lower part of the serpentine sequence $(220-280 \mathrm{~cm})$ contains about $20 \%$ calcite, decreasing to about $10 \%$ between 90 and $180 \mathrm{~cm}$ (Fig. 4). The sediment below and above the serpentine-rich interval is marked by a variation of calcite between $1 \%$ and $25 \%$.

High concentrations of barium and zinc occur between 300 and $360 \mathrm{~cm}$, decreasing into the serpentine (Fig 5). In contrast, chromium content increases at this transition from the lower sediment into the serpentine (Fig. 3).

The boundary between pelagic sediments and the top of the serpentine is marked by sharp changes in concentrations of $\mathrm{MgO}, \mathrm{Ni}, \mathrm{Cr}$, $\mathrm{Ba}$, and $\mathrm{Zn}$ (Figs. 3, 5; Table 2).

Chondrite-normalized REE of the serpentine sequence show a pattern quite different from sediments above and below. The pattern is more like that of MORB. A mixture of $80 \%$ peridotite from Hess Deep and $20 \%$ sediments from the upper part of core 95 results in a pattern similar to that of the serpentine sequence (Fig. 6).

No minerals known from other serpentine sequences, such as brucite, magnetite, talc (Cannat et al., 1992; Boillot et al., 1980; Bonatti et al., 1973; Lonsdale et al., 1980), are found in core 95. Relicts of the unaltered rocks were not identified in this sequence.

The pelagic sediments above and below the serpentine sequence are similar, making up one unit that is interrupted by the serpentines. The pelagic unit is intrinsically heterogeneous and changes in com- position with depth. Minerals identified are calcite in several layers and nontronite, which is consistent with Schmitz et al. (1982), who identified a smectite. Smectite can be formed by temperatures up to $200^{\circ} \mathrm{C}$ by hydrothermal alteration of basalt (Corliss et al., 1978; Mot$\mathrm{tl}$ and Holland, 1978).

Elements such as nickel, manganese, zinc, and arsenic show strongly differing values within the ooze. Profiles of these elements exhibit discrete maxima and minima peaks (Fig. 7). The heterogeneity of this sequence is also shown by major elements such as $\mathrm{Al}_{2} \mathrm{O}_{3}$ and $\mathrm{SiO}_{2}$ (Fig. 8).

The curves reflect steady but varying hydrothermal activity in Hess Deep affecting the chemistry of the sediments. A maxima of hydrothermal activity is evident at $350 \mathrm{~cm}$, where enrichment of arsenic and antimony coincides with high concentrations of iron and manganese at $355 \mathrm{~cm}$. High values of $\mathrm{Cu}$ and $\mathrm{Ni}$ are also found at the manganese maxima. The difference of $5 \mathrm{~cm}$ in the location of the maxima of these hydrothermal elements can be explained by early diagenetic processes. In summary, the curves of these elements (Fig. 7) support the thesis of a hydrothermal source. PGE also show relatively high concentrations at this depth (Table 4).

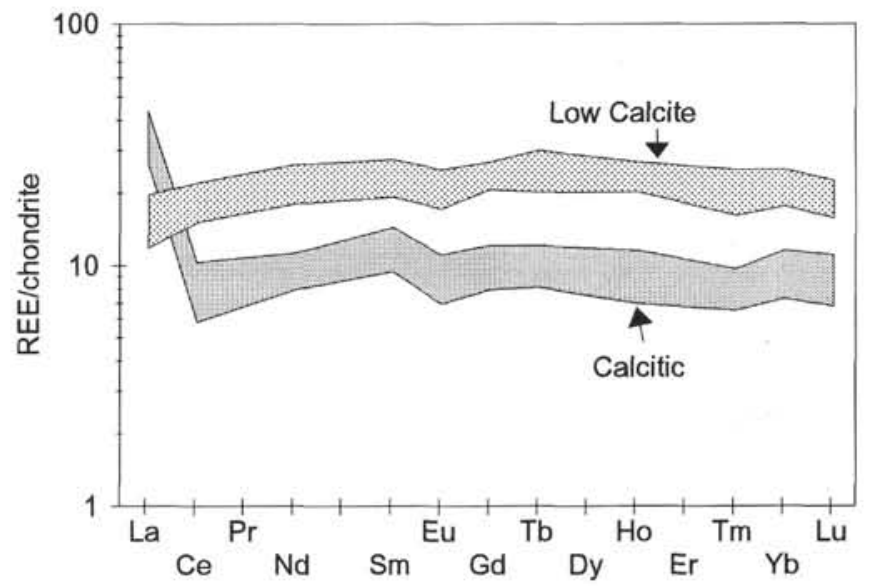

Figure 2. Normalized REE data of sediment samples from Site 894. Normalization against Boynton-chondrite (Boynton, 1984).

Table 1 (continued).

\begin{tabular}{|c|c|c|c|c|c|c|c|c|c|c|c|c|c|}
\hline \multirow[b]{2}{*}{$\begin{array}{l}\text { Core, section, } \\
\text { interval }(\mathrm{cm})\end{array}$} & \multirow[b]{2}{*}{$\begin{array}{l}\text { Depth } \\
\text { (mbsf) }\end{array}$} & \multicolumn{12}{|c|}{ INAA } \\
\hline & & $\begin{array}{l}\mathrm{La} \\
(\mathrm{ppm})\end{array}$ & $\begin{array}{c}\mathrm{Ce} \\
(\mathrm{ppm})\end{array}$ & $\begin{array}{l}\mathrm{Nd} \\
(\mathrm{ppm})\end{array}$ & $\underset{(\mathrm{ppm})}{\mathrm{Sm}}$ & $\begin{array}{c}\text { Eu } \\
(\mathrm{ppm})\end{array}$ & $\begin{array}{c}\mathrm{Gd} \\
(\mathrm{ppm})\end{array}$ & $\begin{array}{l}\mathrm{Tb} \\
(\mathrm{ppm})\end{array}$ & $\begin{array}{l}\mathrm{Ho} \\
(\mathrm{ppm})\end{array}$ & $\begin{array}{l}\mathrm{Tm} \\
(\mathrm{ppm})\end{array}$ & $\begin{array}{c}\mathrm{Yb} \\
(\mathrm{ppm})\end{array}$ & $\begin{array}{l}\mathrm{Lu} \\
(\mathrm{ppm})\end{array}$ & $\begin{array}{c}\mathrm{Hf} \\
(\mathrm{ppm})\end{array}$ \\
\hline \multicolumn{14}{|l|}{$147-894 \mathrm{~A}-$} \\
\hline IR-1, 22-27 & 0.22 & 8.8 & 6.4 & 6.4 & 2.28 & 0.72 & 3 & 0.5 & 0.7 & 0.3 & 2.1 & 0.3 & 0.6 \\
\hline 1R-1, 59-61 & 0.59 & 5.6 & 11.4 & 10.1 & 3.58 & 1.24 & 6 & 1.0 & 1.5 & 0.5 & 3.6 & 0.5 & 2.1 \\
\hline IR-1, 97-99 & 0.97 & 4.1 & 11.6 & 11.6 & 4.21 & 1.43 & 6 & 1.2 & 1.6 & 0.6 & 3.9 & 0.5 & 2.8 \\
\hline $1 \mathrm{R}-1,128-130$ & 1.28 & 4.8 & 12.4 & 12.0 & 4.17 & 1.46 & $<1$ & 1.2 & 1.8 & 0.6 & 4.3 & 0.6 & 2.8 \\
\hline $1 \mathrm{R}-2,0-2$ & 1.50 & 4.5 & 12.3 & 11.4 & 4.18 & 1.48 & $<1$ & 1.1 & $<0.2$ & 0.6 & 4.1 & 0.6 & 2.9 \\
\hline IR-2, 29-31 & 1.79 & 3.8 & 12.1 & 11.8 & 4.14 & 1.44 & 6 & 1.2 & 1.4 & 0.6 & 4.2 & 0.6 & 3.0 \\
\hline IR-2, 61-63 & 2.11 & 4.4 & 13.4 & 13.3 & 4.13 & 1.51 & 5 & 1.2 & $<0.2$ & 0.6 & 4.3 & 0.6 & 3.0 \\
\hline 1R-2, 94-96 & 2.44 & 3.6 & 12.6 & 10.9 & 3.95 & 1.48 & 5 & 1.1 & 1.3 & 0.7 & 4.1 & 0.6 & 2.8 \\
\hline IR $-3,3-5$ & 3.03 & 4.9 & 14.8 & 10.8 & 4.43 & 1.59 & 6 & 1.3 & 1.6 & 0.7 & 4.6 & 0.6 & 3.4 \\
\hline IR-3, 26-28 & 3.26 & 4.6 & 13.2 & 12.4 & 4.53 & 1.59 & 6 & 1.2 & 1.6 & 0.5 & 3.8 & 0.6 & 3.1 \\
\hline $1 \mathrm{R}-3,79-81$ & 3.79 & 3.9 & 13.3 & 11.6 & 4.42 & 1.61 & 6 & 1.2 & $<0.2$ & 0.6 & 4.5 & 0.6 & 3.4 \\
\hline IR-3, 132-134 & 4.32 & 4.5 & 14.0 & 12.6 & 4.45 & 1.49 & 6 & 1.1 & $<0.2$ & 0.5 & 4.6 & 0.6 & 3.4 \\
\hline $1 \mathrm{R}-4,31-33$ & 4.81 & 4.6 & 13.3 & 15.1 & 4.95 & 1.66 & 6 & 1.2 & $<0.2$ & 0.5 & 4.9 & 0.7 & 3.6 \\
\hline IR-4, 79-81 & 5.29 & 4.8 & 13.3 & 12.6 & 4.65 & 1.58 & $<1$ & 1.2 & 1.7 & 0.6 & 4.6 & 0.6 & 3.6 \\
\hline IR-5, 7-9 & 5.57 & 4.3 & 13.5 & 10.8 & 4.29 & 1.49 & $<1$ & 1.1 & 1.7 & $<0.2$ & 4.4 & 0.6 & 3.2 \\
\hline IR-5, 54-56 & 6.04 & 4.0 & 16.6 & 11.9 & 4.45 & 1.51 & 5 & I.1 & 1.5 & 0.5 & 4.5 & 0.6 & 3.4 \\
\hline \multicolumn{14}{|l|}{$147-894 \mathrm{D}$} \\
\hline IR-1, 47-49 & 0.47 & 9.8 & 5.1 & 6.1 & 1.95 & 0.53 & 3 & 0.4 & $<0.2$ & 0.2 & 1.7 & 0.3 & $<0.3$ \\
\hline $1 \mathrm{R}-1,99-101$ & 0.99 & 12.7 & 7.3 & 5.8 & 2.66 & 0.76 & 3 & 0.5 & 0.8 & $<0.2$ & 2.2 & 0.3 & 0.5 \\
\hline \multicolumn{14}{|l|}{$147-894 \mathrm{E}$} \\
\hline $1 \mathrm{R}-1,1-3$ & 0.01 & 7.8 & 5.1 & 5.3 & 1.96 & 0.56 & 2 & 0.5 & $<0.2$ & $<0.2$ & 1.9 & 0.3 & 0.6 \\
\hline IR-1, 50-52 & 0.50 & 9.7 & 4.7 & 5.0 & 1.98 & 0.62 & $<1$ & 0.4 & $<0.2$ & $<0.2$ & 2.0 & 0.3 & 0.6 \\
\hline $1 \mathrm{R}-1,99-101$ & 0.99 & 10.0 & 6.9 & 5.5 & 2.23 & 0.69 & 3 & 0.4 & 0.6 & 0.3 & 2.3 & 0.3 & 1.1 \\
\hline $1 \mathrm{R}-1,148-150$ & 1.48 & 8.7 & 4.5 & 4.9 & 1.79 & 0.50 & 2 & 0.4 & 0.5 & 0.2 & 1.5 & 0.2 & $<0.3$ \\
\hline
\end{tabular}


Table 2. Chemical composition of core 95.

\begin{tabular}{|c|c|c|c|c|c|c|c|c|c|c|c|c|c|}
\hline \multirow[b]{2}{*}{$\begin{array}{c}\text { Depth } \\
\text { (cmbsf) }\end{array}$} & \multicolumn{13}{|c|}{ Major elements } \\
\hline & $\begin{array}{l}\mathrm{SiO}_{2} \\
(\%)\end{array}$ & $\begin{array}{c}\mathrm{TiO}_{2} \\
(\%)\end{array}$ & $\begin{array}{c}\mathrm{Al}_{2} \mathrm{O}_{3} \\
(\%)\end{array}$ & $\begin{array}{c}\mathrm{Fe}_{2} \mathrm{O}_{31 \text { tot }} \\
(\%)\end{array}$ & $\begin{array}{l}\mathrm{MnO} \\
(\%)\end{array}$ & $\begin{array}{l}\mathrm{MgO} \\
(\%)\end{array}$ & $\begin{array}{l}\mathrm{CaO} \\
(\%)\end{array}$ & $\begin{array}{c}\mathrm{Na}_{2} \mathrm{O} \\
(\%)\end{array}$ & $\begin{array}{l}\mathrm{K}_{2} \mathrm{O} \\
(\%)\end{array}$ & $\begin{array}{l}\mathrm{P}_{2} \mathrm{O}_{5} \\
(\%)\end{array}$ & $\begin{array}{l}\text { L.O.1. } \\
(\%)\end{array}$ & $\begin{array}{l}\mathrm{FeO} \\
(\%)\end{array}$ & $\begin{array}{c}\mathrm{Fe}_{2} \mathrm{O}_{3} \\
(\%)\end{array}$ \\
\hline 0 & 48.6 & 0.549 & 6.74 & 13.49 & 1.58 & 15.12 & 3.2 & 0.831 & 0.370 & 0.294 & 9.4 & 2.69 & 11.89 \\
\hline 10 & 49.3 & 0.630 & 7.44 & 14.54 & 0.93 & 13.03 & 3.3 & 1.135 & 0.460 & 0.318 & 8.4 & & \\
\hline 20 & 49.7 & 1.034 & 10.84 & 14.04 & 0.60 & 10.08 & 6.0 & 1.901 & 0.427 & 0.234 & 5.2 & & \\
\hline 30 & 51.5 & 0.786 & 8.58 & 15.14 & 0.69 & 9.71 & 4.1 & 1.545 & 0.548 & 0.341 & 7.3 & 2.57 & 13.49 \\
\hline 40 & 49.8 & 0.661 & 7.92 & 14.21 & 1.32 & 12.38 & 3.7 & 1.296 & 0.456 & 0.282 & 7.7 & & \\
\hline 50 & 47.5 & 0.800 & 7.76 & 15.36 & 1.74 & 10.19 & 5.1 & 1.144 & 0.498 & 0.308 & 8.4 & 2.31 & 14.20 \\
\hline 60 & 35.8 & 0.617 & 5.56 & 11.56 & 0.23 & 7.47 & 18.9 & 0.865 & 0.381 & 0.177 & 17.4 & & \\
\hline 70 & 46.0 & 0.647 & 7.37 & 10.58 & 0.21 & 11.14 & 9.5 & 1.026 & 0.429 & 0.196 & 11.7 & & \\
\hline 80 & 39.9 & 0.578 & 5.92 & 10.80 & 0.22 & 10.76 & 14.4 & 0.841 & 0.434 & 0.179 & 15.2 & 2.45 & 10.02 \\
\hline 90 & 40.2 & 0.314 & 3.32 & 9.25 & 0.30 & 29.37 & 5.2 & 0.333 & 0.099 & 0.077 & 12.7 & & \\
\hline 100 & 39.2 & 0.314 & 3.33 & 8.99 & 0.26 & 29.48 & 5.2 & 0.336 & 0.086 & 0.084 & 12.7 & 1.01 & 9.18 \\
\hline 110 & 37.7 & 0.333 & 3.33 & 9.14 & 0.30 & 29.04 & 6.7 & 0.326 & 0.089 & 0.108 & 13.7 & & \\
\hline 120 & 36.4 & 0.324 & 3.29 & 8.91 & 0.27 & 28.41 & 7.3 & 0.334 & 0.073 & 0.099 & 14.2 & & \\
\hline 130 & 37.2 & 0.349 & 3.42 & 9.34 & 0.25 & 29.51 & 6.3 & 0.287 & 0.074 & 0.084 & 14.1 & 2.34 & 8.27 \\
\hline 140 & 37.5 & 0.349 & 3.45 & 9.31 & 0.26 & 30.33 & 5.5 & 0.317 & 0.071 & 0.083 & 13.9 & & \\
\hline 150 & 36.9 & 0.352 & 3.43 & 9.28 & 0.26 & 30.02 & 5.5 & 0.342 & 0.066 & 0.081 & 14.0 & 2.47 & 8.05 \\
\hline 160 & 37.5 & 0.347 & 3.40 & 9.37 & 0.30 & 30.12 & 5.8 & 0.269 & 0.089 & 0.095 & 13.8 & & \\
\hline 170 & 36.7 & 0.362 & 3.47 & 9.30 & 0.33 & 28.95 & 6.2 & 0.321 & 0.074 & 0.092 & 14.1 & & \\
\hline 180 & 37.0 & 0.353 & 3.47 & 9.33 & 0.43 & 28.49 & 6.4 & 0.335 & 0.071 & 0.115 & 13.8 & & \\
\hline 190 & 37.0 & 0.329 & 3.49 & 9.30 & 0.48 & 27.23 & 7.4 & 0.331 & 0.071 & 0.127 & 14.0 & & \\
\hline 200 & 36.1 & 0.361 & 3.71 & 9.45 & 0.46 & 24.34 & 9.7 & 0.361 & 0.119 & 0.135 & 14.7 & 2.60 & 8.19 \\
\hline 210 & 36.0 & 0.354 & 3.73 & 9.28 & 0.46 & 23.63 & 10.7 & 0.357 & 0.123 & 0.131 & 15.2 & & \\
\hline 220 & 34.8 & 0.390 & 3.87 & 9.64 & 0.49 & 20.58 & 12.7 & 0.429 & 0.174 & 0.153 & 16.2 & & \\
\hline 230 & 34.9 & 0.409 & 3.95 & 9.82 & 0.49 & 19.79 & 12.9 & 0.445 & 0.191 & 0.154 & 16.3 & & \\
\hline 240 & 35.6 & 0.420 & 4.01 & 9.87 & 0.51 & 19.62 & 13.0 & 0.448 & 0.213 & 0.156 & 16.3 & 2.11 & 9.45 \\
\hline 250 & 35.5 & 0.409 & 3.94 & 9.79 & 0.56 & 19.39 & 12.8 & 0.428 & 0.214 & 0,156 & 16.3 & & \\
\hline 260 & 35.8 & 0.445 & 4.09 & 9.96 & 1.02 & 18.65 & 13.0 & 0.486 & 0.255 & 0.167 & 16.3 & & \\
\hline 270 & 35.7 & 0.415 & 3.98 & 9.87 & 0.66 & 18.72 & 12.6 & 0.482 & 0.243 & 0.160 & 16.2 & & \\
\hline 280 & 36.6 & 0.436 & 4.13 & 10.04 & 1.09 & 17.20 & 12.8 & 0.536 & 0.272 & 0.168 & 16.0 & & \\
\hline 290 & 40.5 & 0.513 & 5.61 & 9.91 & 0.55 & 11.92 & 14.3 & 0.861 & 0.386 & 0.179 & 14.9 & & \\
\hline 300 & 54.1 & 0.830 & 7.77 & 12.35 & 0.51 & 7.10 & 6.5 & 1.257 & 0.650 & 0.217 & 7.9 & 2.74 & 10.36 \\
\hline 310 & 40.7 & 0.947 & 7.42 & 11.03 & 0.41 & 5.75 & 17.4 & 1.216 & 0.556 & 0.181 & 13.9 & & \\
\hline 320 & 54.7 & 0.537 & 7.34 & 12.01 & 0.50 & 9.68 & 5.3 & 1.062 & 0.423 & 0.182 & 7.0 & & \\
\hline 330 & 48.7 & 0.676 & 7.82 & 13.18 & 0.34 & 8.08 & 9.0 & 1.265 & 0.545 & 0.262 & 9.5 & 2.81 & 11.44 \\
\hline 340 & 52.8 & 0.616 & 6.83 & 15.22 & 0.59 & 7.68 & 5.6 & 1.118 & 0.565 & 0.362 & 8.4 & & \\
\hline 350 & 48.1 & 0.346 & 3.99 & 17.86 & 1.12 & 5.40 & 7.6 & 0.577 & 0.608 & 1.872 & 11.0 & 1.51 & 18.39 \\
\hline 355 & 39.6 & 0.407 & 4.12 & 8.95 & 2.31 & 9.75 & 15.4 & 0.620 & 0.331 & 0.221 & 17.4 & & \\
\hline 360 & 53.5 & 0.575 & 6.99 & 14.23 & 0.37 & 8.21 & 5.1 & 0.983 & 0.589 & 0.227 & 7.9 & 2.65 & 12.51 \\
\hline
\end{tabular}

Notes: $\mathrm{L} . \mathrm{O} \cdot \mathrm{I} .=$ loss of ignition. INAA $=$ instrumental neutron activation analysis.

Table 2 (continued).

\begin{tabular}{|c|c|c|c|c|c|c|c|c|c|c|}
\hline \multirow[b]{2}{*}{$\begin{array}{c}\text { Depth } \\
\text { (cmbsf) }\end{array}$} & \multicolumn{10}{|c|}{ Minor elements } \\
\hline & $\begin{array}{c}\mathrm{Ni} \\
(\mathrm{ppm})\end{array}$ & $\begin{array}{c}\mathrm{Cu} \\
(\mathrm{ppm})\end{array}$ & $\begin{array}{c}\mathrm{Zn} \\
(\mathrm{ppm})\end{array}$ & $\begin{array}{c}\text { As } \\
\text { (ppm) }\end{array}$ & $\begin{array}{c}\mathrm{Br} \\
(\mathrm{ppm})\end{array}$ & $\begin{array}{c}\mathrm{Rb} \\
(\mathrm{ppm})\end{array}$ & $\begin{array}{c}\mathrm{Sr} \\
(\mathrm{ppm})\end{array}$ & $\begin{array}{c}\mathrm{Y} \\
(\mathrm{ppm})\end{array}$ & $\begin{array}{c}\mathrm{Zr} \\
\text { (ppm) }\end{array}$ & $\begin{array}{c}\mathrm{Pb} \\
(\mathrm{ppm})\end{array}$ \\
\hline 0 & 434 & 397 & 242 & 20.6 & 78 & 7 & 232 & 33 & 70 & 23 \\
\hline 10 & 356 & 419 & 240 & 20.9 & 63 & 8 & 239 & 36 & 75 & 22 \\
\hline 20 & 151 & 302 & 183 & 9.0 & 27 & 9 & 175 & 34 & 86 & 16 \\
\hline 30 & 225 & 368 & 238 & 20.6 & 57 & 10 & 247 & 37 & 90 & 26 \\
\hline 40 & 341 & 357 & 220 & 20.2 & 34 & 8 & 237 & 33 & 77 & 20 \\
\hline 50 & 243 & 369 & 260 & 20.2 & 33 & 10 & 298 & 40 & 93 & 25 \\
\hline 60 & 144 & 209 & 170 & 14.4 & 25 & 10 & 514 & 30 & 68 & 11 \\
\hline 70 & 254 & 459 & 239 & n.d. & 86 & 7 & 333 & 34 & 78 & 18 \\
\hline 80 & 276 & 274 & 191 & 5.9 & 54 & 12 & 458 & 29 & 70 & 19 \\
\hline 90 & 883 & 114 & 105 & 3.8 & 7 & 4 & 372 & 9 & 30 & 7 \\
\hline 100 & 935 & 134 & 104 & 5.1 & 6 & 5 & 367 & 9 & 32 & 7 \\
\hline 110 & 917 & 112 & 106 & 6.9 & 8 & 7 & 615 & 8 & 35 & 10 \\
\hline 120 & 935 & 135 & 112 & 4.1 & 6 & 7 & 771 & 10 & 37 & 7 \\
\hline 130 & 994 & 141 & 116 & 5.2 & 7 & 4 & 496 & 9 & 34 & 7 \\
\hline 140 & 984 & 146 & 114 & 2.6 & 8 & 5 & 370 & 12 & 35 & 9 \\
\hline 150 & 1010 & 141 & 109 & 1.9 & 7 & 6 & 371 & 9 & 35 & 8 \\
\hline 160 & 994 & 151 & 109 & 5.7 & 6 & 5 & 432 & 11 & 33 & 6 \\
\hline 170 & 1073 & 159 & 104 & 3.5 & 9 & 5 & 460 & 10 & 36 & 9 \\
\hline 180 & 1067 & 177 & 107 & 8.7 & 9 & 7 & 467 & 12 & 37 & 9 \\
\hline 190 & 1063 & 235 & 121 & 5.4 & 8 & 5 & 555 & 10 & 34 & 10 \\
\hline 200 & 985 & 277 & 124 & 8.1 & 11 & 5 & 731 & 13 & 39 & 7 \\
\hline 210 & 982 & 299 & 127 & 7.9 & 13 & 7 & 820 & 14 & 38 & 7 \\
\hline 220 & 903 & 293 & 145 & 4.4 & 19 & 10 & 850 & 16 & 48 & 14 \\
\hline 230 & 886 & 288 & 145 & 10.3 & 19 & 9 & 858 & 19 & 49 & 10 \\
\hline 240 & 900 & 291 & 147 & 9.7 & 21 & 10 & 842 & 19 & 49 & 14 \\
\hline 250 & 887 & 294 & 145 & 13.6 & 18 & 8 & 835 & 18 & 50 & 10 \\
\hline 260 & 858 & 251 & 136 & 14.4 & 19 & 8 & 844 & 18 & 49 & 8 \\
\hline 270 & 892 & 275 & 144 & 14.7 & 21 & 11 & 818 & 18 & 50 & 10 \\
\hline 280 & 815 & 260 & 154 & 15.7 & 22 & 9 & 778 & 18 & 50 & 12 \\
\hline 290 & 529 & 335 & 182 & 8.3 & 40 & 8 & 650 & 25 & 56 & 10 \\
\hline 300 & 191 & 284 & 192 & 11.3 & 68 & 9 & 250 & 34 & 86 & 21 \\
\hline 310 & 120 & 152 & 137 & 7.2 & 22 & 11 & 470 & 30 & 89 & 11 \\
\hline 320 & 226 & 316 & 187 & 3.8 & 49 & 7 & 209 & 29 & 60 & 14 \\
\hline 330 & 171 & 264 & 197 & 20.0 & 35 & 10 & 310 & 36 & 79 & 22 \\
\hline 340 & 196 & 301 & 224 & 32.2 & 54 & 10 & 272 & 35 & 81 & 29 \\
\hline 350 & 168 & 228 & 170 & 148.4 & 44 & 11 & 542 & 28 & 55 & 34 \\
\hline 355 & 387 & 414 & 153 & 9.5 & 34 & 6 & 644 & 25 & 53 & 14 \\
\hline 360 & 184 & 337 & 219 & 9.6 & 51 & 10 & 235 & 34 & 76 & 26 \\
\hline
\end{tabular}


Table 2 (continued).

\begin{tabular}{|c|c|c|c|c|c|c|c|c|c|c|c|}
\hline \multirow[b]{2}{*}{$\begin{array}{c}\text { Depth } \\
\text { (cmbsf) }\end{array}$} & \multicolumn{11}{|c|}{ INAA } \\
\hline & $\begin{array}{c}\mathrm{Sc} \\
(\mathrm{ppm})\end{array}$ & $\begin{array}{c}\mathrm{Cr} \\
(\mathrm{ppm})\end{array}$ & $\begin{array}{c}\mathrm{Fe}_{2} \mathrm{O}_{3} \\
(\%)\end{array}$ & $\begin{array}{c}\mathrm{Co} \\
(\mathrm{ppm})\end{array}$ & $\begin{array}{c}\mathrm{Sb} \\
(\mathrm{ppm})\end{array}$ & $\underset{(\mathrm{ppm})}{\mathrm{Cs}}$ & $\begin{array}{c}\mathrm{Ba} \\
(\mathrm{ppm})\end{array}$ & $\begin{array}{c}\mathrm{La} \\
(\mathrm{ppm})\end{array}$ & $\begin{array}{c}\mathrm{Ce} \\
(\mathrm{ppm})\end{array}$ & $\begin{array}{c}\mathrm{Nd} \\
\text { (ppm) }\end{array}$ & $\begin{array}{c}\mathrm{Sm} \\
\text { (ppm) }\end{array}$ \\
\hline 0 & 18 & 311 & 12.67 & 78 & 1.76 & 0.34 & 2523 & 14 & 13 & 14 & 4.0 \\
\hline 10 & 20 & 296 & 13.78 & 71 & 1.54 & 0.21 & 2763 & 16 & 13 & 14 & 4.4 \\
\hline 20 & 32 & 239 & 13.39 & 65 & 0.92 & 0.28 & 1330 & 10 & 13 & 12 & 4.0 \\
\hline 30 & 23 & 222 & 14.18 & 59 & 1.61 & 0.47 & 2917 & 17 & 16 & 17 & 4.9 \\
\hline 40 & 21 & 279 & 13.72 & 88 & 1.49 & 0.49 & 2698 & 15 & 12 & 13 & 4.3 \\
\hline 50 & 22 & 213 & 14.97 & 82 & 2.15 & 0.62 & 3116 & 18 & 15 & 17 & 5.2 \\
\hline 60 & 16 & 169 & 11.78 & 38 & 0.97 & 0.3 & 1815 & 13 & 12 & 12 & 3.8 \\
\hline 70 & 20 & 257 & 10.71 & 64 & 1.85 & 0.24 & 2793 & 16 & 13 & 15 & 4.4 \\
\hline 80 & 17 & 231 & 11.04 & 68 & 1.76 & 0.69 & 2455 & 14 & 12 & 13 & 4.0 \\
\hline 90 & 12 & 600 & 9.09 & 84 & 0.19 & 0.13 & 192 & 3 & 5 & 4 & 1.2 \\
\hline 100 & 12 & 590 & 8.86 & 93 & 0.22 & 0.29 & 221 & 3 & 4 & 2 & 1.2 \\
\hline 110 & 12 & 595 & 9.04 & 89 & 0.24 & 0.25 & 233 & 3 & 3 & 3 & 1.2 \\
\hline 120 & 12 & 594 & 9.12 & 90 & 0.29 & 0.14 & 235 & 3 & 4 & 4 & 1.2 \\
\hline 130 & 12 & 565 & 8.88 & 87 & 0.24 & 0.2 & 248 & 3 & 6 & 3 & 1.2 \\
\hline 140 & 12 & 579 & 8.98 & 91 & 0.19 & 0.18 & 227 & 2 & 5 & 4 & 1.3 \\
\hline 150 & 12 & 583 & 9.11 & 93 & 0.17 & 0.16 & 244 & 3 & 4 & 4 & 1.3 \\
\hline 160 & 12 & 575 & 9.01 & 93 & 0.17 & 0.2 & 246 & 2 & 4 & 2 & 1.3 \\
\hline 170 & 12 & 588 & 9.17 & 98 & 0.4 & 0.2 & 290 & 3 & 4 & 4 & 1.3 \\
\hline 180 & 13 & 598 & 9.32 & 100 & 0.31 & 0 & 322 & 3 & 4 & 3 & 1.4 \\
\hline 190 & 12 & 599 & 9.15 & 105 & 0.37 & 0.25 & 366 & 3 & 4 & 4 & 1.4 \\
\hline 200 & 12 & 538 & 9.2 & 113 & 0.46 & 0 & 168 & 4 & 4 & 5 & 1.7 \\
\hline 210 & 12 & 504 & 9.06 & 115 & 0.49 & 0.13 & 700 & 4 & 5 & 5 & 1.7 \\
\hline 220 & 12 & 404 & 9.45 & 113 & 0.65 & 0.13 & 1100 & 6 & 6 & 5 & 2.2 \\
\hline 230 & 13 & 410 & 9.97 & 118 & 0.55 & 0.12 & 1149 & 7 & 7 & 8 & 2.4 \\
\hline 240 & 13 & 400 & 9.96 & 119 & 0.61 & 0.24 & 1139 & 7 & 7 & 5 & 2.4 \\
\hline 250 & 12 & 374 & 9.47 & 111 & 0.55 & 0.22 & 1089 & 7 & 6 & 7 & 2.2 \\
\hline 260 & 13 & 427 & 9.72 & 108 & 0.49 & 0.16 & 920 & 6 & 7 & 7 & 2.3 \\
\hline 270 & 13 & 393 & 10.01 & 117 & 0.63 & 0.2 & 1257 & 7 & 6 & 8 & 2.5 \\
\hline 280 & 13 & 372 & 9.77 & 106 & 0.66 & 0.15 & 1359 & 8 & 7 & 8 & 2.5 \\
\hline 290 & 15 & 290 & 9.64 & 88 & 1.73 & 0.14 & 2107 & 11 & 11 & 11 & 3.4 \\
\hline 300 & 21 & 165 & 12.54 & 57 & 1.46 & 0.44 & 3182 & 14 & 14 & 14 & 4.8 \\
\hline 310 & 20 & 134 & 11.15 & 41 & 0.94 & 0.38 & 1708 & 10 & 11 & 12 & 4.2 \\
\hline 320 & 22 & 267 & 12.06 & 71 & 1.05 & 0.26 & 3517 & 13 & 11 & 11 & 3.9 \\
\hline 330 & 21 & 188 & 13.22 & 52 & 1.29 & 0.36 & 3181 & 16 & 12 & 13 & 4.7 \\
\hline 340 & 19 & 171 & 15.23 & 61 & 1.27 & 0.4 & 4212 & 17 & 13 & 15 & 4.8 \\
\hline 350 & 11 & 97 & 17.95 & 61 & 4.33 & 0.46 & 3904 & 16 & 12 & 11 & 4.0 \\
\hline 355 & 12 & 198 & 9.14 & 70 & 0.91 & 0.11 & 2806 & 12 & 10 & 11 & 3.2 \\
\hline 360 & 21 & 202 & 14.6 & 63 & 2.82 & 0.53 & 4329 & 16 & 14 & 16 & 4.5 \\
\hline
\end{tabular}

Table 2 (continued).

\begin{tabular}{|c|c|c|c|c|c|c|c|c|c|c|c|}
\hline \multirow[b]{2}{*}{$\begin{array}{l}\text { Depth } \\
\text { (cmbsf) }\end{array}$} & \multicolumn{11}{|c|}{ INAA } \\
\hline & $\begin{array}{c}\text { Eu } \\
(\mathrm{ppm})\end{array}$ & $\begin{array}{c}\text { Gd } \\
(\mathrm{ppm})\end{array}$ & $\begin{array}{c}\mathrm{Tb} \\
(\mathrm{ppm})\end{array}$ & $\begin{array}{c}\text { Ho } \\
\text { (ppm) }\end{array}$ & $\begin{array}{l}\mathrm{Tm} \\
\text { (ppm) }\end{array}$ & $\begin{array}{c}\mathrm{Yb} \\
(\mathrm{ppm})\end{array}$ & $\begin{array}{c}\text { Lu } \\
(\mathrm{ppm})\end{array}$ & $\begin{array}{l}\text { Hf } \\
(\mathrm{ppm})\end{array}$ & $\begin{array}{c}\mathrm{Au} \\
(\mathrm{ppb})\end{array}$ & $\begin{array}{c}\text { Th } \\
\text { (ppm) }\end{array}$ & $\underset{(\mathrm{ppm})}{\mathrm{U}}$ \\
\hline 0 & 1.2 & 4.2 & 0.86 & 1.34 & 0.51 & 3.4 & 0.42 & 1.4 & 13 & 0.43 & 0.77 \\
\hline 10 & 1.4 & 4.7 & 0.94 & 1.23 & 0.72 & 3.7 & 0.49 & 1.3 & 12 & 0.65 & 0.72 \\
\hline 20 & 1.3 & 5.4 & 0.9 & 1.22 & 0.44 & 3.5 & 0.49 & 2.0 & 6 & 0.36 & 0.35 \\
\hline 30 & 1.5 & 5.9 & 1.13 & 1.72 & 0.59 & 4.0 & 0.59 & 1.6 & 10 & 0.59 & 0.64 \\
\hline 40 & 1.3 & 3.9 & 0.98 & 1.23 & 0.56 & 3.6 & 0.5 & 1.4 & 5 & 0.43 & 0.75 \\
\hline 50 & 1.6 & 6.3 & 1.14 & 1.61 & 0.58 & 4.3 & 0.58 & 1.8 & 7 & 0.7 & 0.89 \\
\hline 60 & 1.1 & 3.9 & 0.83 & 1.16 & 0.52 & 3.3 & 0.45 & 1.3 & 15 & 0.35 & 0.9 \\
\hline 70 & 1.3 & 5.3 & 1 & 1.37 & 0.53 & 3.6 & 0.5 & 1.7 & 11 & 0.56 & 2.74 \\
\hline 80 & 1.1 & 4.9 & 0.86 & 1.47 & 0.58 & 3.2 & 0.45 & 1.2 & 12 & 0.34 & 1.44 \\
\hline 90 & 0.3 & 1.9 & 0.26 & 0.36 & 0.18 & 1.1 & 0.14 & 0.8 & 3 & 9.6 & 0.66 \\
\hline 100 & 0.3 & 0.9 & 0.28 & 0.4 & 0.21 & 1.0 & 0.13 & 0.8 & 4 & 10.1 & 0.68 \\
\hline 110 & 0.4 & 0.5 & 0.3 & 0.44 & 0.19 & 1.1 & 0.16 & 0.7 & 6 & 8.7 & 0.78 \\
\hline 120 & 0.4 & 2.7 & 0.29 & 0.46 & 0.1 & 1.2 & 0.14 & 0.7 & 3 & 10 & 0.84 \\
\hline 130 & 0.3 & 0.5 & 0.2 & 0.46 & 0.22 & 1.1 & 0.16 & 0.7 & 1 & 11.6 & 0.82 \\
\hline 140 & 0.4 & 2.0 & 0.28 & 0.31 & 0.21 & 1.2 & 0.15 & 0.6 & 3 & 12.4 & 0.85 \\
\hline 150 & 0.4 & 1.7 & 0.27 & 0.49 & 0.13 & 1.1 & 0.14 & 0.8 & 4 & 11.3 & 0.79 \\
\hline 160 & 0.4 & 1.6 & 0.27 & 0.43 & 0.18 & 1.2 & 0.18 & 0.7 & 5 & 10.2 & 0.86 \\
\hline 170 & 0.4 & 0.6 & 0.32 & 0.6 & 0.14 & 1.1 & 0.16 & 0.8 & 5 & 13.1 & 0.81 \\
\hline 180 & 0.4 & 1.5 & 0.33 & 0.4 & 0.18 & 1.2 & 0.16 & 0.7 & 3 & 10.7 & 0.67 \\
\hline 190 & 0.4 & 1.1 & 0.28 & 0.44 & 0.17 & 1.2 & 0.19 & 0.9 & 6 & 9.3 & 0.98 \\
\hline 200 & 0.5 & 0.8 & 0.27 & 0.5 & 0.2 & 1.4 & 0.16 & 0.9 & 10 & 0.1 & 0.96 \\
\hline 210 & 0.5 & 1.4 & 0.36 & 0.49 & 0.18 & 1.4 & 0.23 & 0.8 & 9 & 0.06 & 0.87 \\
\hline 220 & 0.5 & 1.2 & 0.46 & 0.55 & 0.24 & 1.7 & 0.28 & 0.9 & 9 & 9.2 & 0.92 \\
\hline 230 & 0.7 & 3.6 & 0.46 & 0.73 & 0.25 & 1.9 & 0.27 & 1.0 & 10 & 0.21 & 0.93 \\
\hline 240 & 0.7 & 2.6 & 0.45 & 0.64 & 0.24 & 1.8 & 0.29 & 1.1 & 10 & 12.2 & 0.87 \\
\hline 250 & 0.6 & 0.9 & 0.43 & 0.75 & 0.21 & 1.7 & 0.27 & 0.9 & 9 & 0.07 & 0.88 \\
\hline 260 & 0.7 & 0.5 & 0.5 & 0.64 & 0.31 & 1.8 & 0.28 & 0.8 & 11 & 0.12 & 0.85 \\
\hline 270 & 0.7 & 1.8 & 0.52 & 0.65 & 0.31 & 1.9 & 0.28 & 1.0 & 11 & 0.14 & 0.96 \\
\hline 280 & 0.7 & 2.9 & 0.54 & 0.68 & 0.34 & 2.0 & 0.31 & 0.8 & 8 & 0.15 & 0.74 \\
\hline 290 & 0.9 & 3.4 & 0.68 & 1.04 & 0.43 & 2.6 & 0.4 & 1.1 & 13 & 0.2 & 0.89 \\
\hline 300 & 1.4 & 5.9 & 0.96 & 1.08 & 0.52 & 3.6 & 0.58 & 1.9 & 14 & 0.3 & 0.75 \\
\hline 310 & 1.1 & 3.6 & 0.84 & 1.22 & 0.6 & 3.4 & 0.52 & 1.9 & 3 & 0.32 & 0.91 \\
\hline 320 & 1.2 & 3.3 & 0.77 & 0.97 & 0.43 & 2.7 & 0.43 & 1.0 & 9 & 0.61 & 0.83 \\
\hline 330 & 1.4 & 5.3 & 0.95 & 1.24 & 0.6 & 3.5 & 0.54 & 1.3 & 12 & 0.62 & 0.55 \\
\hline 340 & 1.4 & 5.1 & 0.97 & 1.34 & 0.66 & 3.6 & 0.53 & 1.6 & 14 & 0.5 & 0.5 \\
\hline 350 & 1.1 & 4.7 & 0.75 & 1.2 & 0.47 & 2.8 & 0.46 & 1.1 & 16 & 0.32 & 0.54 \\
\hline 355 & 1.0 & 3.7 & 0.68 & 1.32 & 0.47 & 3.2 & 0.43 & 1.2 & 32 & 0.43 & 2.64 \\
\hline 360 & 1.4 & 5.6 & 1.03 & 1.48 & 0.66 & 4.2 & 0.58 & 1.3 & 33 & 0.64 & 0.4 \\
\hline
\end{tabular}




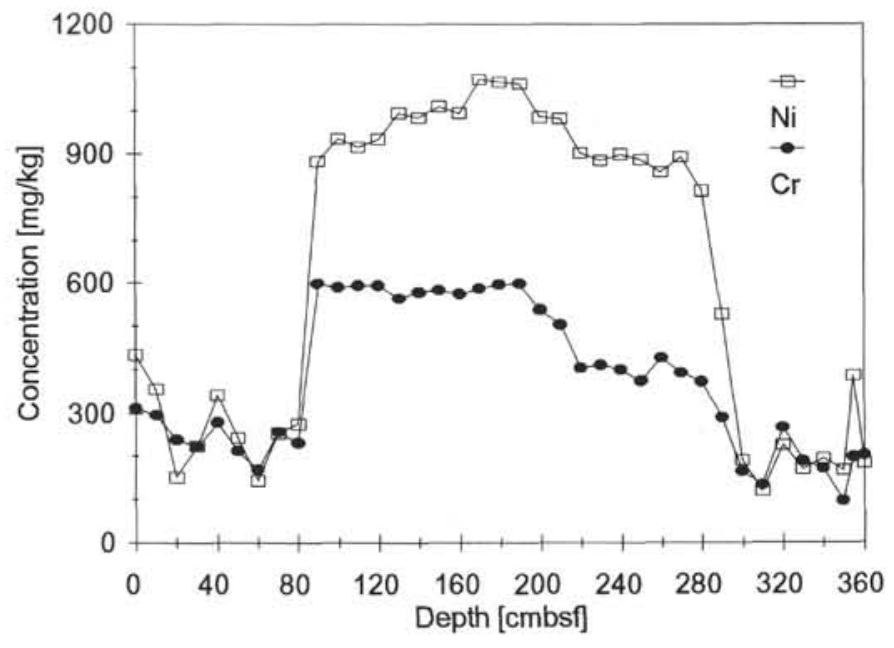

Figure 3. Plot of nickel and chromium concentrations vs. depth in core 95.

Table 3. Difference between the average chemical composition of the serpentine sequence in core 95 and chrysotile.

\begin{tabular}{lccc}
\hline Element \% & $\begin{array}{c}\text { Content in } \\
\text { sample }\end{array}$ & $\begin{array}{c}\text { Content in } \\
\text { chrysotile }\end{array}$ & Difference \\
\hline $\mathrm{SiO}_{2}$ & 37.5 & 30.6 & 6.9 \\
$\mathrm{TiO}_{2}$ & 0.35 & 0.01 & 0.34 \\
$\mathrm{Al}_{2} \mathrm{O}_{3}$ & 3.45 & 0.2 & 3.25 \\
$\mathrm{Fe}_{2} \mathrm{O}_{3}$ & 9.31 & 1 & 8.31 \\
$\mathrm{MnO}$ & 0.26 & 0.03 & 0.23 \\
$\mathrm{MgO}$ & 30.3 & 30.3 & 0 \\
$\mathrm{CaO}$ & 5.5 & 0 & 5.5 \\
$\mathrm{Na} 2$ & 0.32 & 0 & 0.32 \\
$\mathrm{~K}_{2} \mathrm{O}$ & 0.07 & 0 & 0.07 \\
$\mathrm{P}_{2} \mathrm{O}_{5}$ & 0.08 & 0 & 0.08 \\
\hline
\end{tabular}

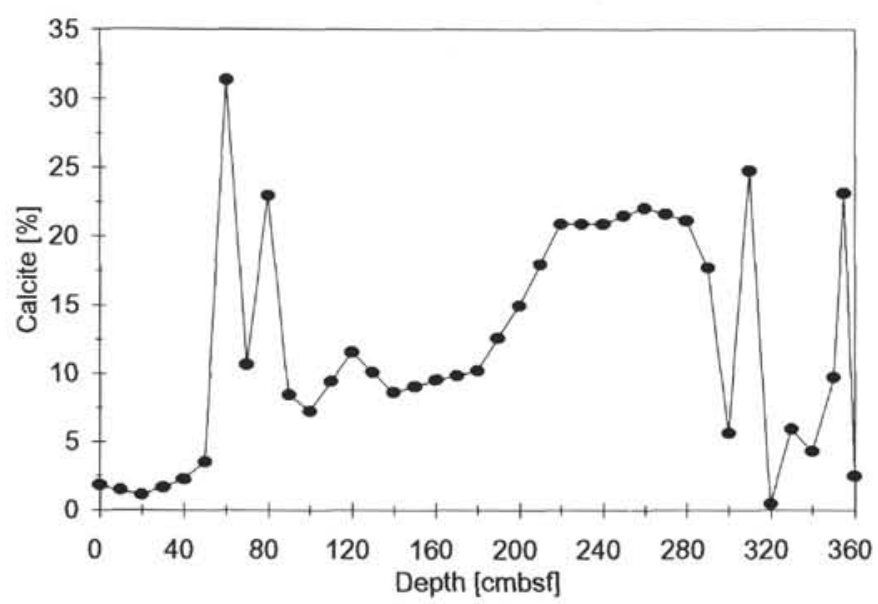

Figure 4. Plot of calcite concentration (wt\%) vs. depth in core 95.

\section{Platinum Group Elements}

In the serpentine and the hydrothermally influenced sequences of core 95, it was possible to determine PGE. The concentrations of the elements are typically less than $10 \mathrm{ppb}$. Concentrations of $\mathrm{Ru}, \mathrm{Ir}$, and $\mathrm{Pt}$ are generally lower in the ooze than in the serpentine sediments, whereas $\mathrm{Pt}$ shows higher values in the upper pelagic sediments. Minima of PGE concentrations, with exception of $\mathrm{Au}$, which has the

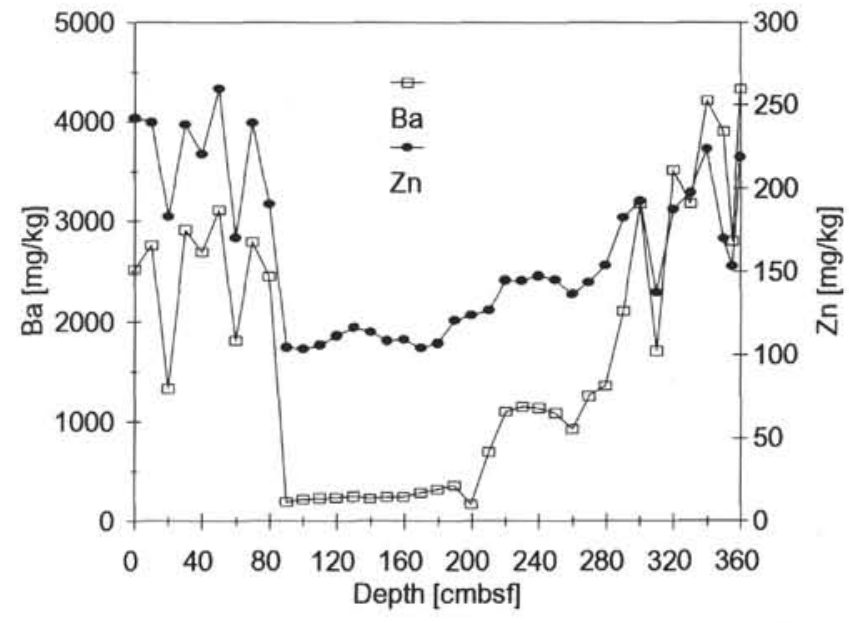

Figure 5. Plot of barium and zinc concentrations vs. depth in core 95 .

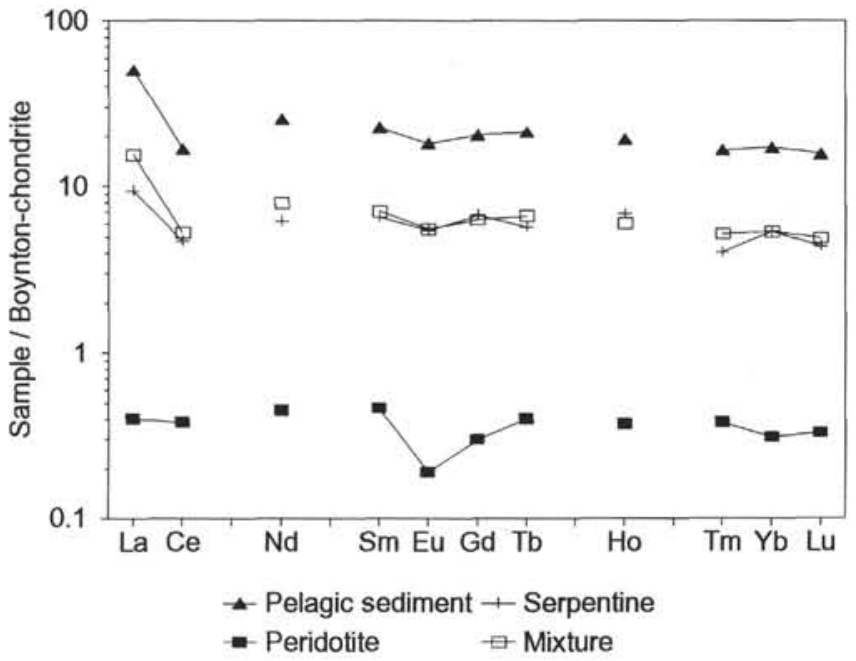

Figure 6. REE patterns of the serpentine sediment sequence and pelagic ooze from core 95 , Hess Deep peridotite, and a reconstructed pattern out of $80 \%$ peridotite and $20 \%$ pelagic ooze, which is the same as the pattern of the serpentine sequence.

highest values, are found in the pelagic sediments. In the serpentines the concentration of all detectable PGE are very constant on a certain level (Table 4). Concentrations of Os, $\mathrm{Rh}$, and $\mathrm{Re}$ in all samples are below detection limit.

Normalization of the PGE in the sediments against the average values of Hess Deep ultramafic rocks shows different patterns for the two types of sediment (Fig. 9). In the serpentines, the PGE are generally depleted relative to peridotites. The PGE show a differentiation in the sequence of $\mathrm{Ir}, \mathrm{Pt}, \mathrm{Ru}, \mathrm{Au}, \mathrm{Pd}$. This order depends not on the descending melting point, which would be $\mathrm{Ir}, \mathrm{Ru}, \mathrm{Pt}, \mathrm{Pd}, \mathrm{Au}$ (Barnes et al., 1985).

The PGE in the hydrothermal sequence are more depleted against peridotite but have the highest enrichments and no fractionation. However, PGE concentrations are much higher than in normal marine pelagic sediments. In most cases, platinum shows the highest depletions and gold the highest enrichments. The event at $350 \mathrm{~cm}$ has a strong enrichment of platinum and iridium. In general, the PGE also show a differentiation in the hydrothermally influenced pelagic sediments of core 95. The sequence is not the same as in the serpentine. $\mathrm{Pt}$ has the highest depletion in normalization against peridotite, followed by $\mathrm{Ir}, \mathrm{Ru}$, and Pd. Au is enriched. 


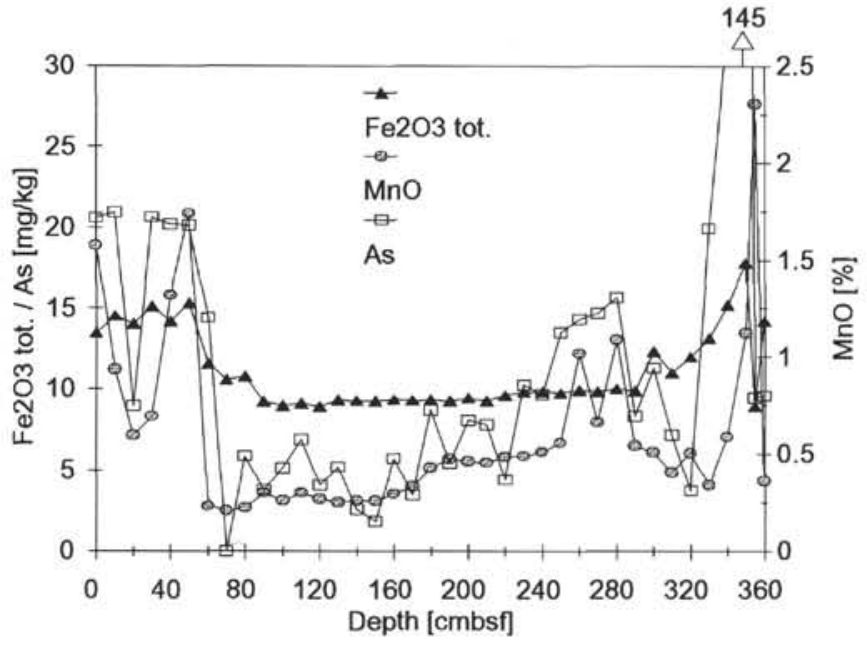

Figure 7. Plot of $\mathrm{Fe}_{2} \mathrm{O}_{3 \text { tot, }}, \mathrm{MnO}$, and As concentrations vs. depth in core 95.

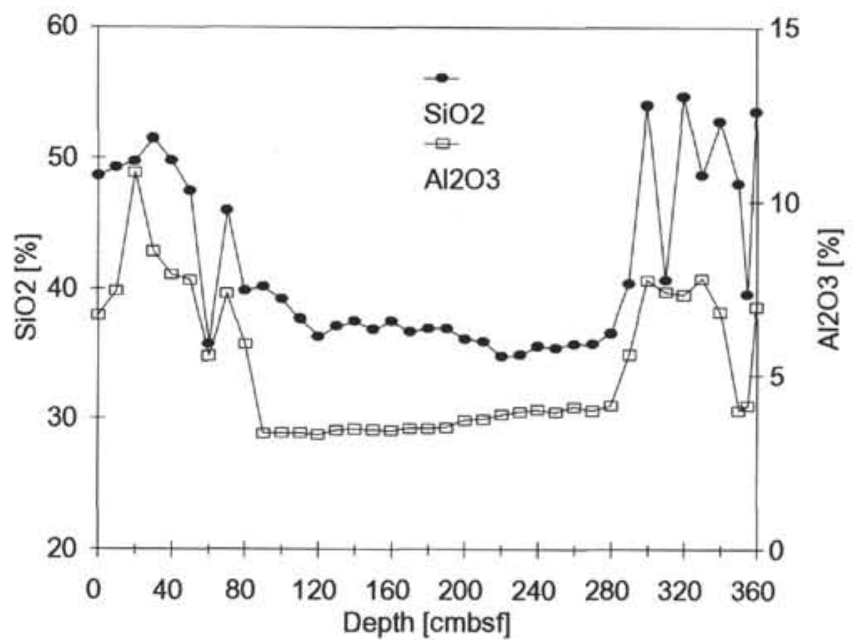

Figure 8. Plot of $\mathrm{SiO}_{2}$ and $\mathrm{Al}_{2} \mathrm{O}_{3}$ concentrations (wt\%) vs. depth in core 95 .

The depletion of PGE must be explained by a process other than the dilution of chrysotile by marine sediments. First, the serpentine sequence consists of about $70 \%$ of chrysotile, and the degree of depletion of PGE is higher. Second, there is fractionation of PGE during depletion. Therefore, PGE must be mobile to a certain level during alteration of peridotites, or are mobilized after alteration by flux of seawater or hydrothermal solutions. The mechanisms of depletion must be different for the serpentines and hydrothermal ooze, because the degree and sequence of depletion of the PGE varies with sediment type.

\section{DISCUSSION}

The sediments in Hess Deep can be categorized on the basis of the geochemical data described in the previous section. Sediments from Site 894 are typically divided into two groups. Foraminiferal oozes and sands containing $55 \%$ to $90 \%$ calcite result from marine sedimentation. They show high $\mathrm{Sr}$ and relatively high $\mathrm{Ba}$ values, probably present as baryte. REE show an enrichment of light REE and a negative $\mathrm{Ce}$ anomaly, which is similar to seawater (Mason and Moore, 1982).

In all three holes the carbonates cover lithic breccias that show the geochemical signature of their source rock. Chondrite-normalized
Table 4. PGE concentrations in samples of core $95[\mu \mathrm{g} / \mathrm{kg}]$.

\begin{tabular}{ccccccccc}
\hline $\begin{array}{c}\text { Depth } \\
(\mathrm{cmbsf})\end{array}$ & $\begin{array}{c}\mathrm{Ru} \\
(\mathrm{ppb})\end{array}$ & $\begin{array}{c}\mathrm{Rh} \\
(\mathrm{ppb})\end{array}$ & $\begin{array}{c}\mathrm{Pd} \\
(\mathrm{ppb})\end{array}$ & $\begin{array}{c}\mathrm{Os} \\
(\mathrm{ppb})\end{array}$ & $\begin{array}{c}\mathrm{Re} \\
(\mathrm{ppb})\end{array}$ & $\begin{array}{c}\mathrm{Ir} \\
(\mathrm{ppb})\end{array}$ & $\begin{array}{c}\mathrm{Pt} \\
(\mathrm{ppb})\end{array}$ & $\begin{array}{c}\mathrm{Au} \\
(\mathrm{ppb})\end{array}$ \\
\hline 10 & 2.6 & $<1$ & 5.8 & $<0.5$ & $<0.1$ & 0.2 & 5.5 & 5.0 \\
20 & 1.6 & $<1$ & 4.5 & $<0.5$ & $<0.1$ & 0.1 & 0.1 & 4.1 \\
40 & 2.6 & $<1$ & 3.1 & $<0.5$ & 0.1 & 0.7 & 2.6 & 1.8 \\
60 & 1.6 & $<1$ & 4.2 & $<0.5$ & $<0.1$ & 0.3 & 1.1 & 8.4 \\
80 & 2.4 & $<1$ & 3.3 & 0.6 & $<0.1$ & 0.7 & 5.5 & 2.7 \\
100 & 3.3 & 5.5 & 6.8 & $<0.5$ & $<0.1$ & 0.7 & 3.0 & 2.2 \\
200 & 1.6 & $<1$ & 3.6 & 0.6 & $<0.1$ & 0.4 & 1.4 & 1.3 \\
250 & 2.9 & $<1$ & 9.5 & 0.6 & $<0.1$ & 0.8 & 2.8 & 4.5 \\
300 & 1.9 & $<1$ & 4.4 & 1.7 & $<0.1$ & 0.4 & 3.9 & 4.1 \\
320 & 1.4 & $<1$ & 4.0 & $<0.5$ & 0.2 & 0.1 & 0.1 & 3.6 \\
340 & 1.6 & $<1$ & 6.2 & $<0.5$ & $<0.1$ & 0.1 & 0.1 & 4.9 \\
350 & 1.4 & $<1$ & 2.6 & 0.8 & $<0.1$ & 0.2 & 0.1 & 1.5 \\
355 & 2.2 & $<1$ & 7.1 & $<0.5$ & $<0.1$ & 0.4 & 1.8 & 6.0 \\
360 & 1.4 & $<1$ & 3.2 & $<0.5$ & $<0.1$ & 0.1 & 0.1 & 3.8 \\
\hline
\end{tabular}

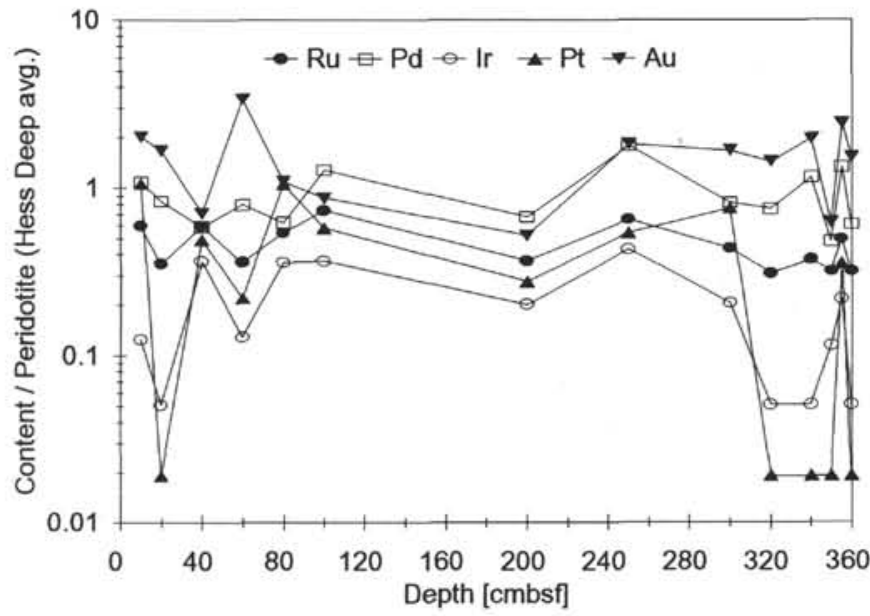

Figure 9. PGE in core 95 normalized against average PGE concentrations in Hess Deep peridotites.

REE patterns show a slight depletion of light and heavy REE, and Eu has a negative anomaly. This is typical for gabbroic rocks. The hypothesis, that these sediments result from pure physical weathering of their underlying rocks, is also supported by the minor element data. In these lithic sediments, there are no hints of marine precipitation expressed by high values of calcite, $\mathrm{Sr}$, or $\mathrm{Ba}$. This, and the sharp boundary between the two sediment types, suggest that the weathering of gabbros occurred in situ and possibly under the calcareous sediment blanket. A single sample in Hole 894A shows a geochemical transition to the overlying foraminiferal sand.

The foraminiferal sands are characterized as turbiditic basin deposits (Shipboard Scientific Party, 1993). Because Site 894 is situated on a morphological high, it must have been lifted in course of its evolution from a basin structure into its present position. For dating this inversion, it would be necessary to obtain, in addition to paleontological data, the relative amount of autochthonous sediment included in and above the foraminiferal turbidites.

If we accept that the foraminiferal sands are not of turbiditic origin, but represent autochthonous sediments, they should have (starting from a thickness of $2 \mathrm{~m}$ and admitting a sedimentation rate of 4 $\mathrm{cm} / \mathrm{k} . \mathrm{y}$.) a minimum age of about 50,000 years. Erosion of sediments by bottom currents would lead to lower sedimentation rates resulting in a higher age for the foraminiferal sands and for the ridge uplift.

In contrast, core 95 is located at the deepest point of the Hess Deep structure, below the CCD. Geochemical data show two different types of sediments in core 95 .

In the median section of the core (from 80 to $290 \mathrm{~cm}$ ) the lithology consists of a serpentine sequence that is mineralogically characterized by chrysotile with some nontronite and calcite. In addition to the 
mineralogical data, the high $\mathrm{Ni}$ and $\mathrm{Cr}$ contents also demonstrate that the source of this material is an altered peridotite. Chromium is probably fixed in relict spinel from peridotite. Harzburgites from Hess Deep have Cr-values of about $2000 \mathrm{mg} / \mathrm{kg}$.

Below and above the serpentine sequence, the sediments are represented by pelagic sediments. Their chemical composition varies widely. Typical hydrothermal elements like $\mathrm{Mn}, \mathrm{Fe}, \mathrm{As}, \mathrm{Ni}$, and $\mathrm{Zn}$ show strong variations, even between neighboring samples. The highest concentrations of these elements are found at about $350 \mathrm{~cm}$, but the highest contents in $\mathrm{Mn}$ and correlated elements $(\mathrm{Ni}, \mathrm{Cu})$ are found at $355 \mathrm{~cm}$. This slight shift is probably caused by early diagenetic processes. Nevertheless, these sediments are certainly influenced by hydrothermal activity, or could even be partly of hydrothermal origin. Nontronite, the main mineral phase identified, is a common product of hydrothermal activity (Corliss et al., 1978; Mottl and Holland, 1978).

The history of the sediments in core 95 can be summed up as follows. The autochthonous sedimentation was originally pelagic, and hydrothermal activity leads to enrichment of specific elements at certain intervals. Pelagic sedimentation was disturbed by turbiditic currents flowing from the surrounding slopes of Hess Deep. We have to suppose that the source region of these currents lies above the CCD, because the sediments contain calcite. In one of the turbidite types, calcite contents of up to $30 \%$ reflect the input of a foraminiferal sand.

The serpentine sequence may be subdivided into two intervals characterized by a transitional change of the calcite content from $20 \%$ to $10 \%$ at $200 \mathrm{~cm}$. This suggests two inflows of different turbiditic material from above the CCD. The time interval between these two episodes must have been short because no autochthonous sedimentation is documented in the transition zone. Trace element contents (e.g., $\mathrm{Ni}, \mathrm{Cr}, \mathrm{Ba}, \mathrm{Zn}$ ) also support this hypothesis.

The base of the serpentine sequence at $280-310 \mathrm{~cm}$ shows a transition to the underlying sediment in the concentration of most elements. The change results from involvement of autochthonous sediments during emplacement of the turbiditic material. As shown by the REE pattern and content, the serpentine sequence probably consists of up to $80 \%$ altered peridotite and $20 \%$ autochthonous pelagic sediment.

It is known from Leg 147 and from Sonne 60 and Nautile dives (Francheteau et al., 1990) that harzburgites and serpentinites are exposed directly north of the deepest point of the Hess Deep. We interpret them as the source rocks for the serpentine sequence in the sediments of core 95. The serpentine sequence does not represent material that altered in place, because no source rock capable of producing chrysotile was observed at the base of the section.

After deposition of the serpentine, above the turbiditic sequence, the previous pelagic sedimentation continued, as indicated by the sharp upper boundary of the serpentine sequence.

\section{SUMMARY}

Different geochemical signatures are observed in the sediments from Hess Deep. The geochemical data not only identify the type of sediment, but also can be used to reconstruct the sedimentation processes.

Sediments from Site 894 can be divided into two types: marine precipitates consisting of calcite, and baryte that overlie unaltered lithic breccias. The latter shows trace-element concentrations and a normalized REE pattern similar to the underlying gabbroic rocks.

The sediments of core 95 from Hess Deep are composed of different sedimentary sequences. Here, autochthonous pelagic sediment was disturbed by calcareous turbidites and was strongly influenced by hydrothermal activity. Moreover, a 2-m-thick serpentinitic turbid- ite, whose source was altered peridotite from above the CCD, is intercalated with the pelagic sediments.

\section{ACKNOWLEDGMENTS}

This work was supplied by the Deutsche Forschungsgemeinschaft. Helpful discussions and reviews improved the manuscript. We thank our colleagues Z. Berner and M. Leosson. Thanks also go to E. v.d. Flier-Keller, an unnamed reviewer, and K. Gillis.

\section{REFERENCES}

Barnes, S.-J., Naldrett, A.J., and Gorton, M.P., 1985. The origin of the fractionation of Platinum-Group Elements in terrestrial magmas. Chem. Geol., 53:303-323.

Boillot, G., Grimaud, S., Mauffret, A., Mougenot, D., Kornprobst, J., Mergoil-Daniel, J., and Torrent, G., 1980. Ocean-continent boundary off the Iberian margin: a serpentinite diapir west of the Galicia Bank. Earth Planet. Sci. Lett., 48:23-34.

Bonatti, E., Honnorez, J., and Gartner, S., Jr., 1973. Sedimentary serpentinites from the Mid-Atlantic Ridge. J. Sediment. Petrol., 43:728-735.

Boynton, W.V., 1984. Cosmochemistry of the rare earth elements: meteorite studies. In Henderson, P. (Ed.), Rare Earth Element Geochemistry: New York (Elsevier), 63-114, Table 3.3.

Cannat, M., Bideau, D., and Bougault, H., 1992. Serpentinized peridotites and gabbros in the Mid-Atlantic Ridge axial valley at $15^{\circ} 37^{\prime} \mathrm{N}$ and 1652'N. Earth Planet. Sci. Lett., 109:87-106.

Corliss, J.B., Lyle, M., Dymond, J., and Crane, K., 1978. The chemistry of hydrothermal mounds near the Galapagos rift. Earth Planet. Sci. Lett., 40:12-24.

Deer, W.A., Howie, R.A., and Zussman, J., 1982. An Introduction to the Rock-Forming Minerals: London (Longman).

Eckhardt, J.-D., 1993. Geochemische Untersuchungen an jungen Sedimenten von der Galapagos Mikroplatte: hydrothermale und stratigraphisch signifikante Signale. Karlsruher Geochem. H., 3.

Evans, B.W., Johannes, W., Oterdoom, H., and Trommsdorff, V., 1976. Stability of chrysotile and antigorite in the serpentine multisystem. Schweiz. Mineral. Petrogr. Mitt., 56:79-93.

Francheteau, J., Armijo, R., Cheminée, J.L., Hekinian, R., Lonsdale, P.F., and Blum, N., 1990. 1 Ma East Pacific Rise oceanic crust and uppermost mantle exposed by rifting in Hess Deep (equatorial Pacific Ocean). Earth Planet. Sci. Lett., 101:281-295.

Horn, D.R., Delach, M.N., and Horn, B.M., 1973. Metal content of ferromanganese deposits of the ocean. In Off. Int. Decade Ocean Explor., Tech. Rep., 4: Washington (Natl. Sci. Found.).

Lonsdale, P.F., Bischoff, J.L., Burns, V.M., Kastner, M., and Sweeney, R.E., 1980. A high-temperature hydrothermal deposit on the seabed at a Gulf of California spreading center. Earth Planet. Sci. Lett., 49:8-20.

Mason, B., and Moore, C.B., 1982. Principles of Geochemistry: Toronto (Wiley).

Moody, J.B., 1976. Serpentinization: a review. Lithos, 9:125-138.

Mottl, M.J., and Holland, H.D., 1978. Chemical exchange during hydrothermal alteration of basalt by seawater, I. Experimental results for major and minor components of seawater. Geochim. Cosmochim. Acta, 42:11031115.

Puchelt, H., 1967. Zur Geochemie des Bariums im exogenen Zyklus. Sitzungsber. Heidelb. Akad. Wiss., Math.-Natwiss. Kl., 4, Abh.

Schmitz, W., Singer, A., Bäcker, H., and Stoffers, P., 1982. Hydrothermal serpentine in a Hess Deep sediment core. Mar. Geol., 46:M17-M26.

Shipboard Scientific Party, 1993. Site 894. In Gillis, K., Mével, C., Allan, J., et al., Proc. ODP, Init. Repts., 147: College Station, TX (Ocean Drilling Program), 45-108.

Date of initial receipt: 8 August 1994

Date of acceptance: 27 April 1995

Ms 147SR-029 